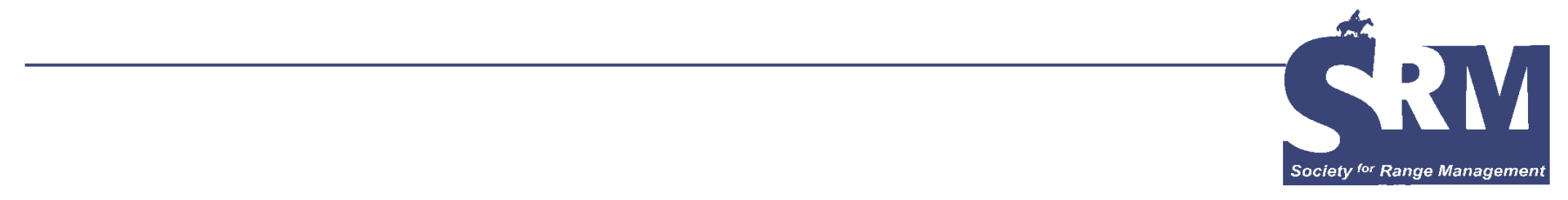

\title{
Rangelands and Climate Change: A Synthesis and Challenges
}

\section{By Joel R. Brown and Jim Thorpe}

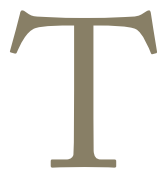

his special issue of Rangelands examines the impacts of climate change on rangeland ecosystems around the globe. The authors have produced a set of readable and highly informative papers that describe both the astounding diversity in rangeland ecosystems around the world and the likely impacts of climate change and possible responses. Without a doubt, the most important challenge will be in terms of the increasing complexity of management within the context of other global forces and conflicts. Although these changes appear overwhelming, we see opportunities for preserving rangeland ecosystems, including the cultures associated with them...if we take the challenge seriously. A big part of taking the challenge seriously is accepting where we are, how we got here, and, finally, what we have to change to survive and thrive.

Much of the history of rangeland management, both as a vocation and a profession, is characterized by principles best described as "conservative, but stable." The key to successful rangeland management is to hold on during stressful periods with the promise of improvement, profits, and progress in better-than-average years. This truism is valid, regardless of whether you are a kangaroo rat caching seeds, a wildebeest herd storing fat, or a rancher building up a bank account. Even a quick scan of history shows this management strategy to be legitimate, and that ignoring these key principles can lead to disaster. Climate change does not alter the basic principles of range management; if anything, it increases their importance, and increases the importance of communicating the applications of those principles. This resilience-based approach will continue to be a rational strategy for managing rangelands in the face of the uncertainty of climate change.
However, the combination of long-term directional shifts, increased short-term variability, and unexpected interactions among factors can easily change the fundamental properties of rangeland ecosystems at rates our current management systems and policies were not designed to accommodate. The real challenge of global change is to respond successfully to the speed of change. Even though the changes in precipitation and temperature appear quite overwhelming when expressed quantitatively and projected over time, they are generally within the range of conditions most current rangeland managers have confronted as episodic events over their lifetime. Drought, heat waves, cold winters, floods, insect and disease outbreaks (and on and on) are the stuff of management. Unfortunately, our history of responding quickly and decisively is not a good one.

Although a great deal of space within each article went toward describing changes in climate and the corresponding changes in rangeland ecosystems, an understanding of these changes is the basis for successful policy development and management response. Improving those policies (and the programs that implement them) and management systems are the focus of this issue. Three themes consistently emerged across all of the articles: the need for improved prediction of changes in climate drivers and the effects on rangeland ecosystems at relevant spatial scales, the importance of integrated monitoring systems, and the need for managers to respond with unprecedented speed and flexibility.

The predictions of the Intergovernmental Panel on Climate Change (IPCC) are the basis for all of the articles in this issue. IPCC efforts to diagnose the causes of climate change, in addition to garnering a Nobel Prize, have been central in communicating and raising the awareness among 
policy makers and the public. As the understanding of the link between human behavior and climate strengthens, the emphasis can shift from explaining observations to prediction. The challenge is to incorporate advances in global scale models into regional short-term models with utility to policy makers, advisors, and managers.

Although models with sufficient precision to be valid at the level of an individual ranch are probably beyond our capability in the immediate future, an increased emphasis on informed interpretation would be of great value. This is not to say that models will not offer that level or precision with basic information (soils, vegetation, climate), but rather that our ability to integrate across scales in a decision-making context will remain a job requiring human interpretive expertise. Providing that expertise will require the collaboration of experts in climatology, ecology, hydrology, production systems, economics, and management, brought together with the express purpose of examining the output of climate models and interpreting the impacts of change on ecosystems, managers, and operating systems at regional and local scales, much as we have tried to do in this issue, but with far greater detail. To be of real value, the time frames of these predictions must be relevant to managers. Communicating these efforts should be a priority (with corresponding resources) and should span the breadth of information and tools available to managers (e.g., ecological site descriptions, extension circulars and brochures, management guidelines and agreements). Although many of these types of tools are currently available (for example, see Climate Assessment for the Southwest: http://www.climas. arizona.edu/), there remains a noticeable lack of consistency, integration, interpretation, and, most importantly, communication.

Predictions, however accurate and accessible, are only valuable for planning. And predictions at large spatial scales and extended time scales can be meaningless or misleading at scales at which management happens. Several of the papers in this issue identified the need to develop and institute "early warning systems," especially for drought. Monitoring, a common theme throughout the rangeland science and management literature, has typically focused on site specific attributes (plant species composition, soil quality indicators, production, cover, etc.). These can be valuable indicators of direction and warnings of impending change, if they are organized into an integrated package that includes information at greater spatial and temporal scales and interpretive standards relative to management objectives. Making good decisions with only site-specific information and devoid of regional trends, landscape context, and interpretive guides is akin to making cross country travel plans with a backyard map.
Improvement in inventory and monitoring methods, and their institutionalization, has been one of the outstanding achievements of rangeland science over the past decade (see http://usda-ars.nmsu.edu/monit_assess/monitoring.php). However, the utility of these tools is limited by an inability to link them to critical regional-, landscape-, and site-level information that can provide a context for their interpretation. Without that context, they lack utility.

Finally, regardless of the accuracy and precision of predictions about climate change and the utilization of monitoring tools, nothing will be implemented unless rangeland managers (ranchers, park mangers, tribes, or homeowners) do something. Climate challenges on rangelands won't occur in isolation from other social, economic, and political challenges that have been posited for the future, especially as the result of anticipated population growth and the subsequent pressure on supporting natural resources and ecosystems. Each of the papers in this issue makes specific reference to a host of factors (population growth, $\mathrm{CO}_{2}$ fertilization, nutrient deposition, invasive species, new operating systems, ecosystem services, etc.) that will interact with climate drivers in ways that either exacerbate or mitigate the direction of change.

This bewildering array of factors and their interactions that drive ecosystem change can easily mask important mileposts that should signal a management response, but important indicators often go unnoticed. The cost of making management changes is often overlooked by scientists and advisors, and land managers are justifiably reluctant to incur costs without a clear indication they are warranted. Inaction on the part of rangeland managers in response to seemingly reliable information is a common theme throughout the short history of our profession. There is a nascent, but growing, effort to better understand why and how people make decisions regarding land management, but this research has been poorly understood and has had little influence on how outreach programs and land management policy are structured. Given the changing ownership of rangeland resources and the resulting diversity of management objectives, an increased emphasis on understanding the decision-making process is critical. Central to this process will be motivating land managers to adopt the management practices that will help them cope with climate change in all its forms.

Authors are Rangeland Scientist, USDA-Natural Resources Conservation Service, Jornada Experimental Range, MSC 3JER, PO Box 30003, New Mexico State University, Las Cruces, NM 88003-0003, USA, joelbrow@nmsu.edu (Brown); and Rancher, Nerwkirk, NM 88431, USA (Thorpe). 